\title{
Load Shedding in Microgrids with Consideration of Voltage Quality Improvement
}

\author{
Bao Long Nguyen Phung \\ Electrical and Electronics Department \\ HCMC University of Technology and Education \\ Ho Chi Minh City, Vietnam \\ 16142018@student.hcmute.edu.vn
}

\author{
Trong Nghia Le \\ Electrical and Electronics Department \\ HCMC University of Technology and Education \\ Ho Chi Minh City, Vietnam \\ trongnghia@hcmute.edu.vn
}

\begin{abstract}
Microgrids have become more and more popular their usefulness as a renewable energy resource has been recognized. The core ability and promise of microgrids is addressing the environmental concerns due to climate change that have been growing during recent years. The innovation of microgrids is that they are designed to operate either in island mode or interconnected with the main grid system. However, when the microgrid operates in islanded mode, faults may occur which can cause system collapse or even blackout. Load curtailment schemes can be utilized to decrease the quantity of associated load to a level that can be securely supported by accessible generation in isolated mode. The main goal of this research is to evaluate the optimal amount of shedding power considering sustainable power sources, with the help of primary and secondary adjustments of the generator to restore the frequency to the allowed range. Particle Swarm Optimization algorithm is applied in this paper to determine the distributed shedding power on each demand load bus which can improve the voltage quality of the isolated microgrid system. The effectiveness of the proposed method is demonstrated through the simulation of IEEE 16- bus microgrid.
\end{abstract}

Keywords-load shedding; islanded microgrid; primary and secondary adjustment; particle swarm optimization

\section{INTRODUCTION}

The imbalance between the power supply and demand loads, when a microgrid (MG) is separated from the main grid by fault or by automatically switching to islanded mode may lead to frequency and voltage droop. In this case, the MG may be overloaded or even collapse and bring the whole system to blackout $[1,2]$. Load shedding schemes can operate to decrease the quantity of associated load to a level that can be securely supported by accessible generation during an emergency. The calculation of the amount of load shedding power is generally based on frequency droop [31. If the calculation is deficient, it will not be able to restore the frequency to a permissible value and will cause over shedding. Researches on load shedding mainly calculate the amount of load shedding power based on the rotation motion of the rotor [4]. However, these strategies do not consider the actual operating conditions such as the primary and secondary control of the generating sets. The techniques of load shedding are divided into three main areas of study [5]: conventional load shedding, adaptive load shedding, and intelligent load shedding. The conventional load shedding is a method of load shedding by using Under Frequency Load Shedding (UFLS) [6], or Under Voltage Load Shedding (UVLS) relays. The UFLS method is known as one of the most used strategies. UFLS technique has a few disadvantages: the load is arbitrarily chosen on the grid when the frequency arrives at the load shedding limit. This technique is not effective when the load shedding areas are spread over the grid and in some cases over shedding or under shedding occurs [7].

Besides the researches on conventional techniques and their development, many intelligent algorithms are used in studying and making new load shedding schemes, for example fuzzy logic, Genetic Algorithm (GA), Artificial Neutral Networks (ANNs), Analytic Hierarchy Priority (AHP), Particle Swarm Optimization (PSO), etc. The fuzzy logic based load shedding scenario is efficient in restoring the frequency of an islanded MG system quickly [6, 8-12]. In some researches, fuzzy - AHP logic algorithm was used in load shedding strategies to measure the weight of load nodes in the system in various load levels [13]. The ANN-based load curtailment method can optimize the amount of load shedding in order to stabilize the power system [16]. A load curtailment based on a combination between ANN and neuro-fuzzy was proposed in [15]. The results show that the ANN can compute the total amount of active power that needs to be shed but it does not determine the number of loads or the distributed load shedding power that need to be shed in each demand load. Authors in $[16,17]$ proposed a load shedding method based on GA gain that can be used in the calculation of the optimal amount of shedding power. In fact, the GA can help in making the calculation speed for load shedding scenario robust, however the limitation of this algorithm is that it cannot help in optimal distributed load shedding for each demand load bus. The efficiency of PSO has been applied in load shedding techniques in [15, 18]. The studies show that PSO can forecast the frequency droop and optimize the amount of the required load demand. However, in this strategy, there's still no limitation in the optimal shedding power. In [19], an UFLS technique based on PSO was studied, and the main objective was to calculate the optimal shedding power in order to stabilize the system frequency. However, the proposed method did not consider the voltage quality criteria in the system. Despite the fact that there are many studies that 
show the effectiveness of PSO in optimal load curtailment scheme, most of the methods are used in general or islanded grid systems, not on islanded MGs with renewable sources.

The main objective of this paper is to plan an optimal load shedding strategy for islanded MGs with renewable energy sources during the islanded operation. The minimum shedding power will be computed based on the ability of primary control of the generators' governor and the reserve power of the generators for the secondary adjustment of the generators. The application of PSO algorithm will determine the optimal distributed shedding power of each load bus. And from that, it can optimize the voltage droop and improve the voltage quality. The effectiveness of this method is tested by the simulations of the IEEE 16-bus system, and the results are compared with a conventional under-frequency load-shedding scheme. Calculated and simulated results showed that the proposed method has less amount of shedding power than the UFLS relay method. Furthermore, the recovery of frequency was still held within the allowable values and maintained the stability of the power system. The current research can be used as a reference for MG system operators and electrical engineering graduate students when considering the issue of optimizing load shedding in the MG system when there is a disconnection from the main grid.

\section{THEORETICAL BACKGROUND}

\section{A. Primary and Secondary Frequency Adjustment}

Under ordinary conditions and in the case of power losses in the framework, the power balance of the MG is introduced by:

$$
P_{\text {main_grid }}+P_{G i}+P_{G_{-} \text {storage }}+P_{R E S}=\sum P_{L j}
$$

where $P_{\text {main grid }}$ is the power from the bulk grid, $P_{G i}$ is the power of the $i^{\text {th }}$ generator that has a speeder or a distributed generator with Auto Frequency Controller (AFC), $\sum P_{L j}$ is the real power of the $j^{\text {th }}$ load in normal operation, and $P_{G_{-}}$storage and $P_{R E S}$ are the capacity of energy storage system and power of Renewable Energy Sources (RES). When the MG disconnects from the main grid, the first reaction of the system will be the primary frequency adjustment. It's a reaction of a power source that has a speeder or an AFC. These frequency controllers will increase the power of generators in relation to the frequency $[20,21]$.

In Figure 1, we see the characteristics of the generators under normal operation before and after the connection loss of the MG from the main grid. Characteristic lines (F), (G) illustrate the characteristic of the load power in the normal operating conditions and during load shedding respectively. Characteristic lines (B), (D) show the output power of a generator without a governor corresponding to the normal condition, after disconnection from the main grid. The (A), (C) and (E) characteristics are the power. $P G_{n}$ and $P G_{n-1}$ are the total value of the output power in the normal operating conditions and during the outage generator respectively, $f_{0}$ is the rated frequency in the normal operating conditions, $f_{l}$ is the frequency of the MG during the islanded operation (in the case that the generators have a governor and the distributed generators have a droop controller), $f_{l}$ ' is the frequency of the outage generator (in case of the generator without a governor), $f_{2}$ is the frequency of the system after the primary and secondary control, and $f_{\text {allowed }}$ is the restored frequency $(59.7 \mathrm{~Hz}$ for power grids with a rated frequency of $60 \mathrm{~Hz}$ ).

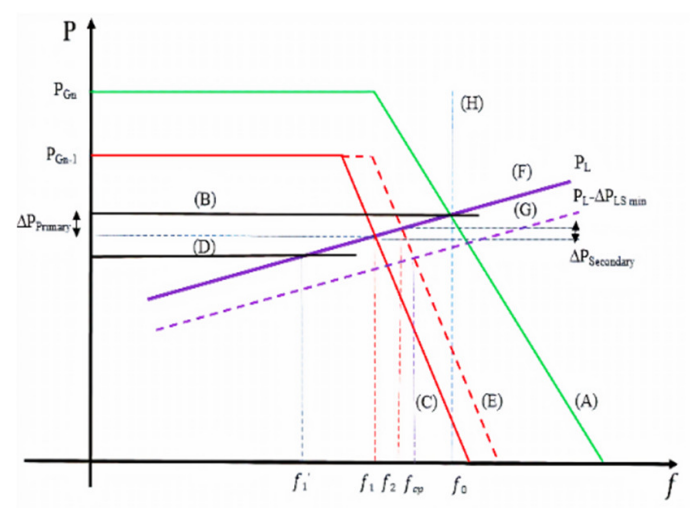

Fig. 1. The relationship between frequency and output power deviation.

When the primary adjustment process for the power source is initiated, the response of the load to the frequency change is also performed. In addition, the RESs that respond slowly to frequency changes are considered not capable of adjusting the primary and secondary process, so they can be considered as negative loads. Equation (1) can be rewritten as:

$$
\begin{gathered}
P_{G i}+\Delta P_{\text {primary }}= \\
\sum P_{L j}-P_{\text {main_grid }}-\left(P_{G_{\text {_storage }}}+P_{R E S}\right)-\Delta P_{L(\text { freq })}
\end{gathered}
$$

where: $\Delta P_{\text {primary }}=\sum_{i=1}^{n-1} \frac{-\Delta f}{R_{i}}$ is the amount of primary control power of the system when fault occurs. In this case it will be the islanded operation mode of the MG. $\Delta f$ is the frequency droop when the MG turns to islanded operation. $R_{i}$ is the ratio between the frequency and generator's power. It also represents the adjustment of the speed of slip. $\Delta P_{L(\text { freq })}$ is the load whose power changes with the frequency.

If the frequency will not go back to the allowed range after the primary adjustment process, the secondary adjustment will proceed. The secondary adjustment process is the process of the primary adjustment made through the effect of the Automatic Generating Center (AGC) system on a number of specified units, or a dispatch command $[20,21]$. The balance power equation will be rewritten as:

$$
\begin{gathered}
P_{G i}+\Delta P_{\text {primary }}+\Delta P_{\text {sec ondary }}= \\
\sum P_{L j}-P_{\text {main_grid }}-\left(P_{G_{-} \text {storage }}+P_{R E S}\right)-\Delta P_{L(\text { freq })}
\end{gathered}
$$

where $\Delta P_{\text {sec ondary }}=P_{G n, i}-\Delta P_{\text {primary }}$ is the maximum secondary power adjustment of the generator.

\section{B. Particle Swarm Optimization}

PSO was first studied in 1995 [22]. In PSO, the expression "population" alludes to the particles which are dependent upon the best method of conduct. These particles can run in two 
modes: stochastic mode and deterministic mode. Mathematically, the searching process can be presented by simple equations using the position vector $X_{i}=\left[x_{i 1}, x_{i 2}, \ldots ., x_{i n}\right]$ and the velocity vector $V_{i}=\left[v_{i 1}, v_{i 2}, \ldots, v_{i n}\right]$ according to the specific dimension of the searching space. In addition, the optimum of the solution in the PSO algorithm depends on the particle position and velocity update by the following equations:

$$
\begin{gathered}
V_{i}^{k+1}=w \cdot V_{i}^{k}+c_{1} r_{1}\left[X_{\text {pbest }}^{k}-X_{i}^{k}\right]+c_{2} r_{2}\left[X_{\text {gbest }}^{k}-X_{i}^{k}\right] \\
X_{i}^{k+1}=X_{i}^{k}+V_{i}^{k+1}
\end{gathered}
$$

where $V_{i}^{\mathrm{k}}, X_{i}^{\mathrm{k}}$ represent the velocity and position of each particle $i$ at iteration $k, w$ is the inertia constant, ranging usually between 0 and $1, c_{1}$ and $c_{2}$ are cognitive coefficients, ranging from 0 to $2, r_{1}$ and $r_{2}$ are random values generated for each velocity update, and $X_{p b e s t}^{k}, X_{\text {gbest }}^{k}$ are the global best positions earned in the swarm's experience and the best position of each particle respectively.

Each part of (4) is defined as: $w \cdot V_{i}^{k}$ is the inertia component, which is responsible for keeping the particle search in the same direction, $c_{1.1}\left[X_{\text {pbest }}^{k}-X_{i}^{k}\right]$ is the cognitive component representing the particle's memory. The cognitive coefficient $c_{l}$ affects the step size of the particle to move toward its local best position $X_{\text {pbest }} \cdot c_{2} r_{2}\left[X_{\text {gbest }}^{k}-X_{i}^{k}\right]$ is the social component. It represents the movement of the particle towards the best region found by the swarm so far. The $c_{2}$ factor is known as the social coefficient, and it also controls the step size of the particle to find the global best positon $X_{\text {gbest }}$. Equation (5) presents the situation of every individual particle by utilizing the new speed and its past position. In other words, a looking through cycle is re-initiated in order to locate the global ideal arrangement. The cycle rehashes itself until it meets one completion criterion, e.g. maximum number of repetitions.

\section{THE PROPOSED LOAD SHEDDING METHOD}

\section{Construction of the Formula of Optimal Amount of Shedding Power}

When the primary and the secondary adjustment do not recover the frequency back to nominal stage, then load shedding is required. In that case, the power balance equation will be:

$$
\begin{gathered}
P_{G i}+\Delta P_{p \text { rimary }}+\Delta P_{\text {sec ondary }} \\
=\sum P_{L j}-P_{\text {main_grid }}-\left(P_{G_{-} \text {storage }}+P_{R E S}\right) \\
-\Delta P_{L(\text { freq })}-\Delta P_{\text {shed_min }} \\
\Leftrightarrow P_{G i}+\sum_{i=1}^{n-1-\Delta f_{\text {allowed }}}+\Delta P_{\text {sec ondary }} \\
=\sum P_{L j}-P_{\text {main_grid }}-\left(P_{G_{-}}\right. \text {storage } \\
\left.-D .(-\Delta \omega)-\Delta P_{\text {shed_min }}\right)
\end{gathered}
$$

where $\Delta f_{\text {allowed }}=f_{n}-f_{\text {droop }}$ is the allowed drop of the frequency, $\Delta P_{\text {shed_min }}$ is the minimum amount of load shed that has the ability to bring the frequency close to the nominal value, $D$ is the characteristic factor change of load in percentage frequency change, ranging between $1 \%$ and $2 \%$. The factor $D$ is experimentally determined, e.g. if $D=2 \%$, then if the frequency changes by $1 \%$, it will lead to the change of loads by $2 \%$. From the above equation, the minimum amount of load shedding can be determined as:

$$
\begin{gathered}
\Delta P_{\text {shed_min }}= \\
{\left[\sum P_{L j}-P_{\text {main_grid }}-\left(P_{G_{-} \text {storage }}+P_{R E S}\right)-D \cdot(-\Delta \omega)\right]} \\
-\left(P_{G i}+\sum_{i=1}^{n-1-\Delta f_{\text {allowed }}}+\Delta P_{i} \sec \text { ondary }\right)
\end{gathered}
$$

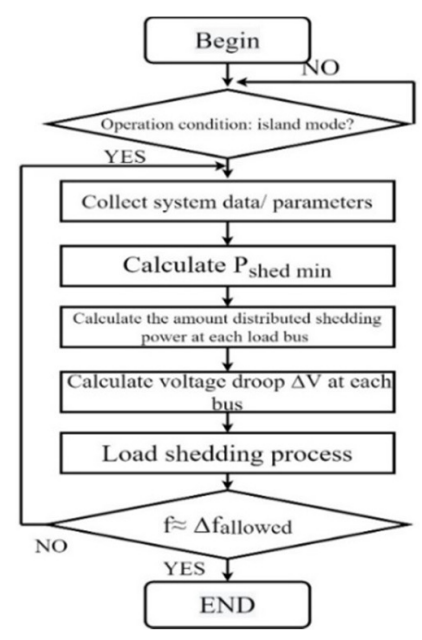

Fig. 2. Proposed load shedding method.

The load shedding method is illustrated in Figure 2. The first step will be to determine all the necessary parameters of the system, including the parameters of loads and generators. Then the operation mode of the system will be considered as well. The next step to calculate the frequency droop, then primary and secondary power adjustment if necessary. After that, the minimum amount of load shedding and the amount of load shed at each bus will be determined by the PSO algorithm. After the amount of load shed is calculated, the load shedding process will be operated. And finally, the overall check must be processed to ensure the system is safe and secure.

\section{Construction of the Objective Function for PSO}

The objective function in the PSO algorithm is based on the voltage quality on each load bus. The main purpose of this function is to find the optimal amount of load shedding on each load bus if the MG turns into islanded mode in order to get the best result of voltage droop. This optimized load shedding scheme not only prevents the system from blacking out when the disconnection occurs, it also provides an improved way to shed load that avoids unnecessary loss. To obtain the objective function or voltage droop control function, the Jacobian matrix will be applied because of its resolution which is finding the amplitude of voltage and phase angle based on the real and 
reactive power in an electrical system. The basic formula of the Jacobian Matrix is shown below:

$$
\left[\begin{array}{l}
\Delta P_{1} \\
\vdots \\
\Delta P_{n-1} \\
\Delta Q_{1} \\
\vdots \\
\Delta Q_{n-1}
\end{array}\right]=\left[\begin{array}{cccccc}
\frac{\partial P_{1}}{\partial \delta_{1}} & \cdots & \frac{\partial P_{1}}{\partial \delta_{n-1}} & \frac{\partial P_{1}}{\partial|V|_{1}} & \cdots & \frac{\partial P_{1}}{\partial|V|_{n-m}} \\
\vdots & \ddots & \vdots & \vdots & \ddots & \vdots \\
\frac{\partial P_{n-1}}{\partial \delta_{1}} & \cdots & \frac{\partial P_{n-1}}{\partial \delta_{n-1}} & \frac{\partial P_{n-1}}{\partial|V|_{1}} & \cdots & \frac{\partial P_{n-1}}{\partial|V|_{n-m}} \\
\frac{\partial Q_{1}}{\partial \delta_{1}} & \cdots & \frac{\partial Q_{1}}{\partial \delta_{n-1}} & \frac{\partial Q_{1}}{\partial|V|_{1}} & \cdots & \frac{\partial Q_{1}}{\partial|V|_{n-m}} \\
\vdots & \ddots & \vdots & \vdots & \ddots & \vdots \\
\frac{\partial Q_{n-1}}{\partial \delta_{1}} & \cdots & \frac{\partial Q_{n-1}}{\partial \delta_{n-1}} & \frac{\partial Q_{n-1}}{\partial|V|_{1}} & \cdots & \frac{\partial Q_{n-1}}{\partial|V|_{n-m}}
\end{array}\right) \times\left[\begin{array}{c}
\Delta \delta_{1} \\
\vdots \\
\Delta \delta_{n-1} \\
|V|_{1} \\
\vdots \\
|V|_{n-m}
\end{array}\right]
$$

where $n$ is the number of nodes in the grid, $m$ is the number of generators in the system, and $n-m$ represents the loads in the electrical network.

By converting (9) and ignoring the 1st generator from the bulk grid in the system because it will be removed after the islanded operation occurs, (10) can be claimed. Besides, in order to achieve the voltage droop equation, the $J 2$ and $J 4$ elements of the Jacobian matrix will be measured:

$$
\left[\begin{array}{c}
\Delta \delta_{2} \\
\vdots \\
\Delta \delta_{n} \\
|V|_{2} \\
\vdots \\
|V|_{n}
\end{array}\right]=\left[\begin{array}{cccccc}
\frac{\partial P_{2}}{\partial \delta_{2}} & \cdots & \frac{\partial P_{2}}{\partial \delta_{n}} & \frac{\partial P_{2}}{\partial|V|_{2}} & \cdots & \frac{\partial P_{2}}{\partial|V|_{n}} \\
\vdots & \ddots & \vdots & \vdots & \ddots & \vdots \\
\frac{\partial P_{n-2}}{\partial \delta_{2}} & \cdots & \frac{\partial P_{n-2}}{\partial \delta_{n}} & \frac{\partial P_{n}}{\partial|V|_{2}} & \cdots & \frac{\partial P_{n}}{\partial|V|_{n}} \\
\frac{\partial Q_{2}}{\partial \delta_{2}} & \cdots & \frac{\partial Q_{2}}{\partial \delta_{n}} & \frac{\partial Q_{2}}{\left.\partial\right|_{2}} & \cdots & \frac{\partial Q_{2}}{\partial|V|_{n}} \\
\vdots & \ddots & \vdots & \vdots & \ddots & \vdots \\
\frac{\partial Q_{n-2}}{\partial \delta_{2}} & \cdots & \frac{\partial Q_{n-2}}{\partial \delta_{n}} & \frac{\partial Q_{n}}{\partial|V|_{2}} & \cdots & \frac{\partial Q_{n}}{\partial|V|_{n}}
\end{array}\right]^{-1} \times\left[\begin{array}{c}
\Delta P_{2} \\
\vdots \\
\Delta P_{n} \\
\Delta Q_{2} \\
\vdots \\
\Delta Q_{n}
\end{array}\right]
$$
bus:

The final function will be the voltage droop control on each

$$
\Delta|V|_{i}=\Delta P_{i} \times J_{2}^{-1}+\Delta Q_{i} \times J_{4}^{-1}
$$

According to the power electrical triangle, $\Delta Q_{i}=\Delta P_{i} \times \tan \varphi$, so (12) can be written :

$$
\Delta|V|_{i}=\Delta P_{i} \times J_{2}^{-1}+\Delta P_{i} \times \tan \varphi \times J_{4}^{-1}
$$

where $\left|V_{i}\right|$ is the voltage droop on each bus, $\Delta P_{i}$ is the real power on the bus, $J_{2}^{-1}$ and $J_{4}^{-1}$ are the inverse $J_{2}, J_{4}$ elements of the Jacobian matrix, and $\tan \varphi$ the phase angle $(\tan \varphi=0.8)$. From (12), the detailed voltage droop equation can be obtained:

$$
\begin{aligned}
& \Delta|V|_{i}= J_{2(i, 2)}^{-1} \times \Delta P_{2}+\ldots .+J_{2(i, m)}^{-1} \times \Delta P_{m} \\
&+J_{2(i, m+1)}^{-1} \times \Delta P_{m+1}+\ldots . . .+J_{2(i, n)}^{-1} \times \Delta P_{n} \\
&+J_{4(i, n+1)}^{-1} \times \Delta P_{2} \times \tan \varphi+\ldots \ldots \\
&+ J_{4(i, 2 n-2)}^{-1} \times \Delta P_{n} \times \tan \varphi
\end{aligned}
$$

In load shedding, the voltage droop will be enormous, and that is one of the most critical dangers of such schemes. This will make the system unstable and cause the whole system to collapse. There are still limitations in the control of that droop, therefore, the first thing that the objective function must do is to find the maximum value that can occur when the voltage falls, then from all the maximum values it must call out the least in the aforesaid index by using the PSO algorithm to make sure that the system is safe and secure after the disconnection. So, the objective function for PSO will be expressed as:

$$
\min \left(\max \left(\Delta|V|_{i}\right)\right)=\min \left(\max \left(\Delta P_{i} \times J_{2}^{-1}+\Delta P_{i} \times \tan \varphi \times J_{4}^{-1}\right)\right.
$$

This algorithm has two main constrictions:

- The amount of shedding power in each load must not be larger than its base power: $0<\Delta P<P_{\text {base }}$. The base power of each load is the power that must not be shed, for example, $P_{\text {base }}=30 \% P_{\text {load }}$. This constraint helps in optimizing the amount of load shed in each load in order to avoid unnecessary loss and prevent overshedding.

- The sum of all the shedding powers of each load is equal or larger than the minimum load shedding power: $\Sigma \Delta P \geq \Delta P_{\text {shed_min }}$ The constraint makes sure the frequency droop is in control and the amount of shedding power is not under-shed.

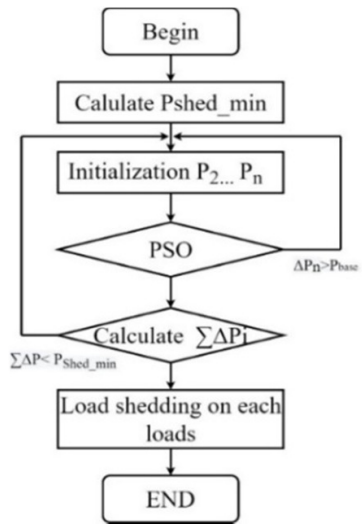

Fig. 3. Flowchart of the PSO algorithm for load shedding.

The steps of the PSO algorithm are shown in Figure 3. The first step in the PSO process is to modify the value of shedding power in each load, in other words, setting the value of $P_{\text {base. }}$ In this case, the value of $P_{\text {base }}$ will be $30 \%$ of each load power. In the index of the voltage droop that has been calculated, the maximum value will be taken. The process will do over the initialization step and find the maximum value again and again, until the maximum value is unchanged after 2 or 3 iterations. The value of the formula $\min \left(\max \left(\Delta\left|V_{i}\right|\right)\right)$, which is calculated by the PSO algorithm, is the optimal value of shedding power in each load.

\section{EXPERIMENTS AND RESULTS}

The MG system chosen to simulate the effectiveness of the proposed method consisted of 16 buses and 6 sources [23], including a transformer at bus 16 which is considered as the 
main, connected to the MG at bus-1, 2 Diesel generator sources, 1 PV panel source, 1 source of wind energy (wind turbine), and a storing energy system which in this case is batteries. When the system goes into islanded mode, the diesel generator at bus-2 is considered as the balancing node. The linear diagram of the system is shown in Figure 4 and the parameters of the sources and the loads are shown in Tables I and II respectively. The system was simulated in PowerWorld Simulation 2019. The bus connected to the main grid is bus-1, which had a fault that caused the MG system to lose connection with the main grid and was forced to operate in islanded mode.

TABLE I. PARAMETERS OF POWER SOURCES IN THE MG SYSTEM

\begin{tabular}{|c|c|c|c|}
\hline Sources & $\boldsymbol{P}_{G}(\mathbf{M W})$ & $\boldsymbol{P}_{G_{n}, \boldsymbol{I}}(\mathbf{M W})$ & $\boldsymbol{R}$ \\
\hline Main grid & 3.87 & & \\
\hline Diesel generator (bus2) & 5.00 & 6.00 & 0.05 \\
\hline Diesel generator (bus8) & 0.90 & 1.0 & 0.05 \\
\hline Battery (bus 11) & 0.3 & 0.5 & 0.00 \\
\hline PV panels (bus 14) & 0.5 & 1.00 & 0.00 \\
\hline Wind turbine (bus 15) & 2.00 & 3.00 & 0.00 \\
\hline
\end{tabular}

TABLE II. PARAMETER OF LOADS IN THE MG SYSTEM

\begin{tabular}{|c|c|}
\hline Loads & Power (MW) \\
\hline Load 3 & 1.06 \\
\hline Load 4 & 1.53 \\
\hline Load 5 & 1.95 \\
\hline Load 7 & 1.17 \\
\hline Load 9 & 1.81 \\
\hline Load 10 & 2.09 \\
\hline Load 12 & 1.2 \\
\hline Load 13 & 1.17 \\
\hline Total load power & 11.98 \\
\hline
\end{tabular}

\section{E. Calculating the Minimum Shedding Power}

In this experiment, two Diesel generators will participate in the frequency adjustment. Applying (2) and the parameters of generators and loads of Table I and II, the frequency droop after the MG disconnection from the grid will be:

$$
\begin{gathered}
5+0.9+\frac{6 \times(-\Delta f)}{0.05 \times 60}+\frac{-\Delta f}{0.05 \times 60}= \\
11.98-0.3-2-\frac{11.98 \times 0.02 \times(-\Delta f)}{60} \Rightarrow \\
\Delta f=-1.4033 \mathrm{~Hz} \Rightarrow \\
f_{\text {droop }}=f_{n}+\Delta f=60-1.4033=58.596 \mathrm{~Hz}
\end{gathered}
$$

Figure 5 shows that the frequency has fell below the safety range after the disconnection. That incident would make the system instable and lead to black out. So, the primary and secondary frequency adjustments need to be processed in order to restore the frequency back to its nominal stage. In this experiment, the primary frequency adjustment will be automatically operated by the speeder due to the reaction of the turbine. The amount of primary control power of the system will be:

$$
\sum_{i=1}^{n} \Delta P_{\text {primary }}=\frac{-6}{0.05} \times \frac{-0.3}{60}+\frac{-1}{0.05} \times \frac{-0.3}{60}=0.7 \mathrm{MW}
$$

The amount of maximum secondary power adjustment of the Diesel generator at bus- 2 is:

$$
\Delta P_{\text {sec ondary }}=6-5-0.6=0.4 \mathrm{MW}
$$

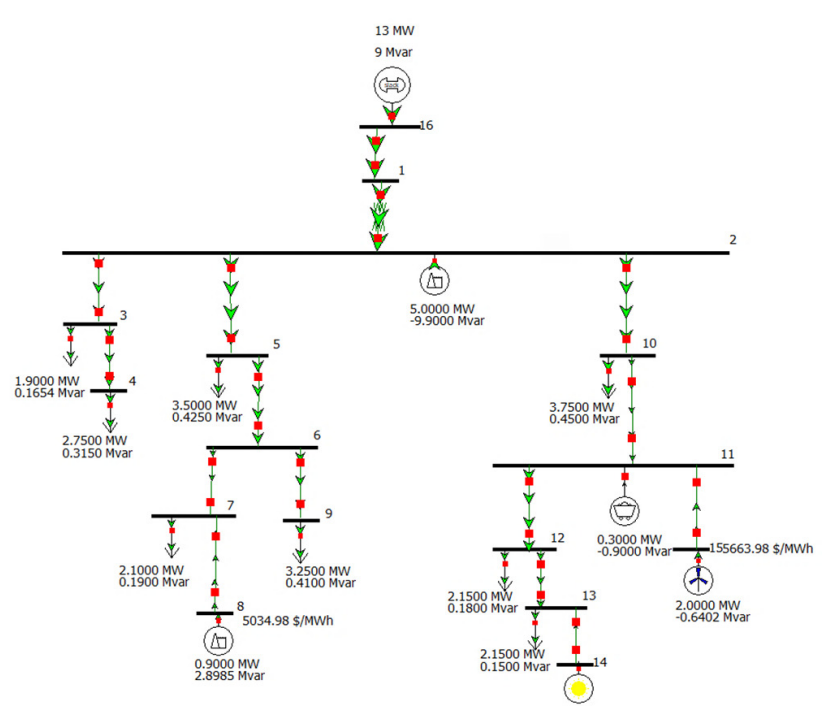

Fig. 4. Linear diagram of the MG model.

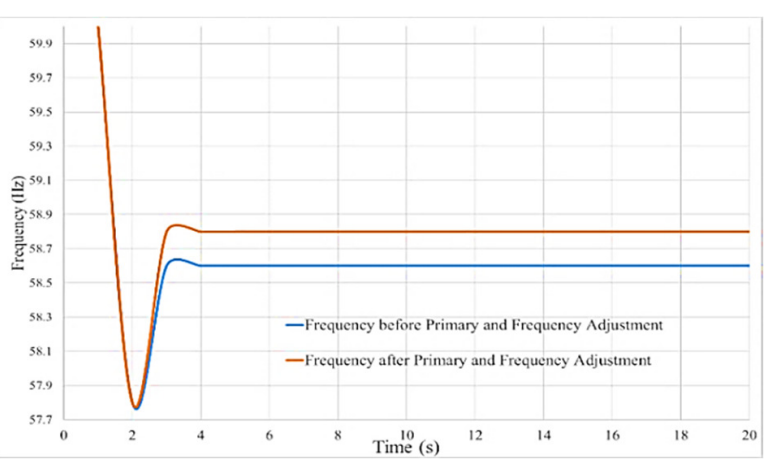

Fig. 5. System frequency after the primary and secondary adjustments.

The frequency has been recovered thanks to the adjustment process, however, it is still under the allowed range, and that could still harm the system. Therefore, load shedding must be applied in order to restore the frequency to normal. Equation (8) is applied to compute the minimum shedding power:

$$
\begin{gathered}
\Delta P_{\text {shed_min }}=11.98-0.3-2-0.5 \\
+\frac{11.98 \times 0.02 \times(-0.3)}{60}-5-0.9-\frac{-6 \times(-0.3)}{0.05 \times 60} \\
-\frac{-1 \times(-0.3)}{0.05 \times 60}-0.4=2.1788 \mathrm{MW}
\end{gathered}
$$

To prove the effectiveness of the proposed method, the load shedding method using UFLS relay is used for comparison. The process of UFLS is implemented when the frequency drops below the frequency setting threshold [24]. The load is usually shed step-by-step based on the load shedding table that is pre-designed based on overall principles and administrator experience. The table manages the amount of load that should 
be shed at each step depending on the decrease of frequency. These values are shown in Table III.

TABLE III. 4 STAGES OF THE UFLS SCHEME

\begin{tabular}{|c|c|c|}
\hline Stage & Frequency $(\mathbf{H z})$ & Cumulative amount of load $\boldsymbol{P}_{\text {shed }}(\mathbf{\%})$ \\
\hline I & 59 & 35 \\
\hline II & 58.8 & 30 \\
\hline III & 58.4 & 20 \\
\hline IV & 58 & 15 \\
\hline
\end{tabular}

The frequency droop is $58.596 \mathrm{~Hz}$, so the amount of load shedding will be the $30 \%$ of total load:

$$
\Delta P_{\text {shed_UFLS }}=0.3 \times 11.98=3.594 \mathrm{MW}
$$

The result shows that the proposed load shedding method has better optimal amount of shedding power than the UFLS method. The frequency comparison between the proposed method and UFLS is illustrated in Figure 6.

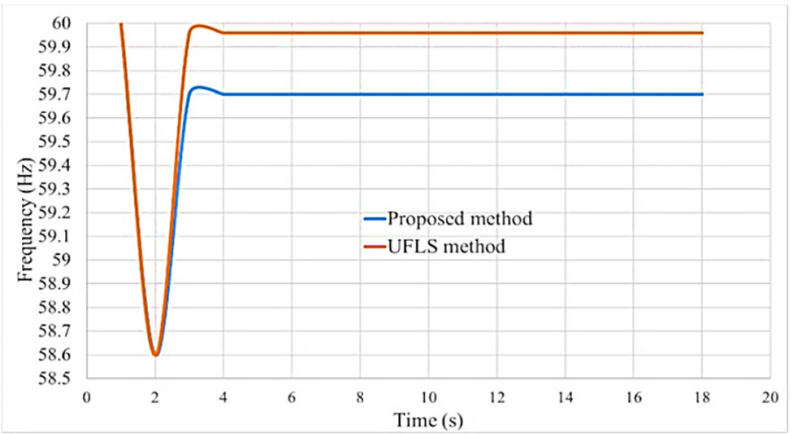

Fig. 6. Comparison of the frequency between the proposed method and UFLS.

The recovery frequency of the proposed method is $59.7 \mathrm{~Hz}$ in comparison with the $59.96 \mathrm{~Hz}$ of the UFLS method. The comparison result shows that although the recovery frequency of the proposed recovery method is not as good as the UFLS method, it is still in the permissible range while it reduces the amount of shedding power. The shedding power is $1.4152 \mathrm{MW}$ lower than that of the traditional UFLS method.

\section{F. Applying PSO to Compute the Optimal Distributed Load Shedding on Each Bus Load}

The restriction of the amount of shedding power that can be shed in each load bus will be considered as the $P_{\text {base }}$. The upper bound and the lower bound of the PSO calculation in this experiment will be: $P_{\text {base }}=30 \% P_{\text {load }}$.

TABLE IV. PARAMETERS OF BASE POWER OF LOADS

\begin{tabular}{|c|c|c|}
\hline Loads & Load power $(\mathbf{M W})$ & $\boldsymbol{P}_{\text {base }}(\mathbf{M W})$ \\
\hline Load 3 & 1.06 & 0.318 \\
\hline Load 4 & 1.53 & 0.462 \\
\hline Load 5 & 1.95 & 0.585 \\
\hline Load 7 & 1.17 & 0.351 \\
\hline Load 9 & 1.81 & 0.543 \\
\hline Load 10 & 2.09 & 0.627 \\
\hline Load 12 & 1.2 & 0.36 \\
\hline Load 13 & 1.17 & 0.351 \\
\hline
\end{tabular}

Using (14) to calculate the index of minimum voltage droop and optimal shedding power on each load bus and by using the comparison by percent, the best result is chosen for the distributed load shedding for each load bus, as shown in Table V.

TABLE V. OPTIMAL SHEDDING POWER ON EACH LOAD

\begin{tabular}{|c|c|}
\hline Loads & Shedding power (MW) \\
\hline Load 3 & 0.185 \\
\hline Load 4 & 0.24623 \\
\hline Load 5 & 0.22319 \\
\hline Load 7 & 0.2125 \\
\hline Load 9 & 0.48086 \\
\hline Load 10 & 0.33978 \\
\hline Load 12 & 0.26464 \\
\hline Load 13 & 0.22673 \\
\hline Total shedding power & 2.1788 \\
\hline
\end{tabular}
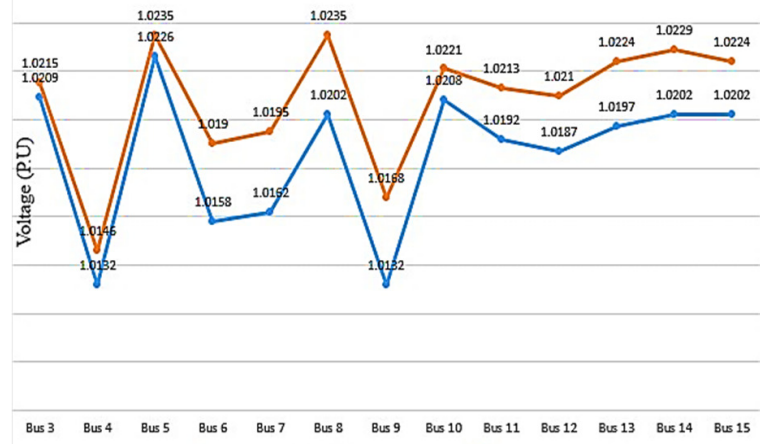

$$
\text { -Before Load Shedding AAfter Load Shedding }
$$

Fig. 7. Voltage droop of the MG before and after load shedding.

Figure 7 shows that the voltage quality has been improved after applying the proposed load shedding strategy. In addition, the result of PSO algorithm (Table V) confirms the proposed method of determining the optimum amount of shedding power on each load bus in order to limit the damage when load shedding is processed while still meeting the technical requirements of the operating specifications of the MG system.

\section{CONCLUSION}

In this paper, an optimal shedding method for MGs in islanded operation with consideration of sustainable power sources has been proposed. The proposed method has recovered the frequency back to the normal stage to maintain the stability of the islanded MG system, based on its ability of adjusting the primary and secondary generators in order to calculate the minimum shedding power and restore the frequency of the system. The effectiveness of the proposed method has been demonstrated on the IEEE 16-bus system. Its performance is better than that of the conventional UFLS method. The result shows that the proposed method can reduce the amount of shedding power while satisfying the technical operating conditions of the network. The current research also applies the PSO algorithm to optimize the amount of distributed shedding power of each load bus in order to improve voltage quality and to prevent damage during the islanded operation. In the future, this project can be developed in several ways, for instance: 
- Multi-objective load shedding can be considered, i.e. combining other algorithms like AHP, fuzzy logic, ANNs or the variants of PSO in order to determine the way in optimizing several issues including load shedding, economic dispatch, etc.

- More research can be conducted in real time cases in order to get better results and solutions that can be used in real systems.

- Applying AI in solving the problem.

\section{REFERENCES}

[1] Z. Zhao, P. Yang, J. M. Guerrero, Z. Xu, and T. C. Green, "MultipleTime-Scales Hierarchical Frequency Stability Control Strategy of Medium-Voltage Isolated Microgrid," IEEE Transactions on Power Electronics, vol. 31, no. 8, pp. 5974-5991, Aug. 2016, https://doi.org/ 10.1109/TPEL.2015.2496869.

[2] H. Gao, Y. Chen, Y. Xu, and C. Liu, "Dynamic load shedding for an islanded microgrid with limited generation resources," Transmission Distribution IET Generation, vol. 10, no. 12, pp. 2953-2961, 2016, https://doi.org/10.1049/iet-gtd.2015.1452.

[3] D. L. H. Aik, "A general-order system frequency response model incorporating load shedding: analytic modeling and applications," IEEE Transactions on Power Systems, vol. 21, no. 2, pp. 709-717, May 2006, https://doi.org/10.1109/TPWRS.2006.873123.

[4] V. V. Terzija, "Adaptive underfrequency load shedding based on the magnitude of the disturbance estimation," IEEE Transactions on Power Systems, vol. 21, no. 3, pp. 1260-1266, Aug. 2006, https://doi.org/ 10.1109/TPWRS.2006.879315.

[5] J. A. Laghari, H. Mokhlis, A. H. A. Bakar, and H. Mohamad, "Application of computational intelligence techniques for load shedding in power systems: A review," Energy Conversion and Management, vol. 75 , pp. 130-140, Nov. 2013, https://doi.org/10.1016/j.enconman. 2013.06.010.

[6] M. Lagouir, A. Badri, and Y. Sayouti, "Optimal power flow management strategies of MicroGrid, using Petri Nets and fuzzy logic approaches," in 2018 4th International Conference on Optimization and Applications (ICOA), Mohammedia, Morocco, Apr. 2018, https://doi.org/10.1109/ICOA.2018.8370517.

[7] J. Tang, J. Liu, F. Ponci, and A. Monti, "Adaptive load shedding based on combined frequency and voltage stability assessment using synchrophasor measurements," IEEE Transactions on Power Systems, vol. 28, no. 2, pp. 2035-2047, May 2013, https://doi.org/10.1109/ TPWRS.2013.2241794.

[8] A. A. Sallam and A. M. Khafaga, "Fuzzy expert system using load shedding for voltage instability control," in LESCOPE'02. 2002 Large Engineering Systems Conference on Power Engineering. Conference Proceedings, Halifax, Canada, Jun. 2002, pp. 125-132, https://doi.org/ 10.1109/LESCPE.2002.1020678.

[9] R. Khezri, S. Golshannavaz, R. Vakili, and B. Memar-Esfahani, "Multilayer fuzzy-based under-frequency load shedding in back-pressure smart industrial microgrids," Energy, vol. 132, pp. 96-105, Aug. 2017, https://doi.org/10.1016/j.energy.2017.05.059.

[10] B. de Nadai N., A. C. Z. de Souza, J. A. da S. Neto, J. G. de C. Costa, F. M. Portelinha, and D. Marujo, "An OffLine Fuzzy-Based DecisionMaking to Load Shedding in Microgrids," in 2019 IEEE PES Innovative Smart Grid Technologies Conference - Latin America (ISGT Latin America), Gramado, Brazil, Sep. 2019, https://doi.org/10.1109/ISGTLA.2019.8895409.

[11] B. Bahmani-Firouzi, E. Farjah, and R. Azizipanah-Abarghooee, "An efficient scenario-based and fuzzy self-adaptive learning particle swarm optimization approach for dynamic economic emission dispatch considering load and wind power uncertainties," Energy, vol. 50, pp. 232-244, Feb. 2013, https://doi.org/10.1016/j.energy.2012.11.017.

[12] P. D. Chung, "Retaining of Frequency in Micro-grid with Wind Turbine and Diesel Generator," Engineering, Technology \& Applied Science
Research, vol. 8, no. 6, pp. 3646-3651, Dec. 2018, https://doi.org/ 10.48084/etasr.2413.

[13] T. N. Le, H. A. Quyen, and N. A. Nguyen, "Application of fuzzyanalytic hierarchy process algorithm and fuzzy load profile for load shedding in power systems," International Journal of Electrical Power \& Energy Systems, vol. 77, pp. 178-184, May 2016, https://doi.org/ 10.1016/j.ijepes.2015.11.044.

[14] R. Hooshmand and M. Moazzami, "Optimal design of adaptive under frequency load shedding using artificial neural networks in isolated power system," International Journal of Electrical Power \& Energy Systems, vol. 42, no. 1, pp. 220-228, Nov. 2012, https://doi.org/ 10.1016/j.ijepes.2012.04.021.

[15] F. Conteh et al., "An effective Load shedding technique for micro-grids using artificial neural network and adaptive neuro-fuzzy inference system," AIMS Energy, vol. 5, no. 5, pp. 814-837, 2017, https://doi.org/10.3934/energy.2017.5.814.

[16] Y. Astriani, G. Shafiullah, and F. Shahnia, "Optimizing Under-voltage Load-shedding Using Genetic Algorithm in Microgrid," in 2019 2nd International Conference on High Voltage Engineering and Power Systems (ICHVEPS), Oct. 2019, pp. 1-6, https://doi.org/10.1109/ ICHVEPS47643.2019.9011109.

[17] C. Chen, W.-T. Tsai, H. Chen, C.-Y. Lee, C.-J. Chen, and H.-W. Lan, "Optimal load shedding planning with genetic algorithm," in 2011 IEEE Industry Applications Society Annual Meeting, Orlando, FL, USA, Oct. 2011, https://doi.org/10.1109/IAS.2011.6074299.

[18] F. He, Y. Wang, K. W. Chan, Y. Zhang, and S. Mei, "Optimal load shedding strategy based on particle swarm optimization," in 8th International Conference on Advances in Power System Control, Operation and Management (APSCOM 2009), Hong Kong, China, Nov. 2009, https://doi.org/10.1049/cp.2009.1795.

[19] N. Sadati, T. Amraee, and A. M. Ranjbar, "A global Particle SwarmBased-Simulated Annealing Optimization technique for under-voltage load shedding problem," Applied Soft Computing, vol. 9, no. 2, pp. 652657, Mar. 2009, https://doi.org/10.1016/j.asoc.2008.09.005.

[20] A. J. Wood, B. F. Wollenberg, and G. B. Sheblé, Power Generation, Operation, and Control, 3rd ed. Hoboken, NJ, USA: Wiley-Interscience, 2013.

[21] K. Soleimani and J. Mazloum, "Designing a GA-Based Robust Controller For Load Frequency Control (LFC)," Engineering, Technology \& Applied Science Research, vol. 8, no. 2, pp. 2633-2639, Apr. 2018, https://doi.org/10.48084/etasr.1592.

[22] J. Kennedy and R. Eberhart, "Particle swarm optimization," in ICNN'95International Conference on Neural Networks, 1995, vol. 4, pp. 1942 1948.

[23] W. Shi, X. Xie, C. Chu, and R. Gadh, "Distributed Optimal Energy Management in Microgrids," IEEE Transactions on Smart Grid, vol. 6, no. 3, pp. 1137-1146, May 2015, https://doi.org/10.1109/TSG. 2014.2373150.

[24] Q. Zhou, Z. Li, Q. Wu, and M. Shahidehpour, "Two-Stage Load Shedding for Secondary Control in Hierarchical Operation of Islanded Microgrids," IEEE Transactions on Smart Grid, vol. 10, no. 3, pp. 3103-3111, May 2019, https://doi.org/10.1109/TSG.2018.2817738. 